\title{
Pandemic COVID-19 and Its Implications in Dentistry: Overt Actions to Deal with Covert Threat
}

\author{
Sumit Munjal ${ }^{1} \odot$, Seema Munjal ${ }^{2}$
}

\begin{abstract}
Background: Currently, transmission routes are still to be determined, but common ones include direct transmission (cough, sneeze, and droplet inhalation transmission) and contact transmission (contact with oral, nasal, and eye mucous membranes). Due to the characteristics of dental settings, the risk of cross infections may be high between dental practitioners and patients.

Aims and objectives: This review aimed at presenting a recent update on the severe acute respiratory syndrome coronavirus 2 (SARS-CoV-2) (COVID-19) with regards to prevention and control. In the unlikely event of providing dental care to suspected or confirmed cases of the new pandemic, dentists must be cognizant of dire precautions.

Materials and methods: A thorough literature search was carried out electronically between 2019 and 2020 present using appropriate keywords. The results were reviewed and prioritized, and the findings were compiled. Out of a total of 94 publications strategically obtained, 23 studies were included in the end for review.

Results: Patients are managed according to the severity of the virulence, and all the possible drug trials have been instigated. The menace being new, the limitations of the study do exist. Vaccines are the most effective strategy since they are more cost-effective than the treatment. Conclusion: Identifying a suspected case of COVID-19 is the only way out to mitigate the spread of this novel infection in dental institutions. Clinical significance: Dental health-care providers ought to keep updated in all respects as we are among the healthcare workers bearing the brunt of the situation.

Keywords: COVID-19 pandemic, Dentistry in COVID-19, Management, Viral infection and dentist.

Journal of Oral Health and Community Dentistry (2021): 10.5005/jp-journals-10062-0102
\end{abstract}

\section{INTRODUCTION}

Pandemics are the large-scale outbreaks of infectious diseases increasing morbidity and mortality over a wide geographic area. Owing to increased travel, urbanization, changes in land use, and greater exploitation of the natural environment, the likelihood of pandemics has increased over the past century. ${ }^{1,2}$ The severe acute respiratory syndrome coronavirus 2 (SARS-CoV-2) (COVID-19) has lately been the sword of Damocles hanging over dentistry. Coronaviruses are large, enveloped, positive-strand RNA viruses that can be divided into four genera: alpha, beta, delta, and gamma, of which alpha and beta CoVs are known to infect humans.

While the coronavirus is de facto mildly severe, the betacoronavirus infections resulted in higher mortality rates. ${ }^{3-7}$ The medico-dental community is ever-increasingly roped in to deliver impregnable innovations and cures, even more so when people are stranded amid pandemics. The cognate threat of contagion to the community itself while delivering treatments is irrefutable, at the same time. Dental settings invariably carry the risk due to the specificity of their procedures, involving faceto-face communication with patients, and frequent exposure to saliva, blood, and other body fluids, and the handling of sharp instruments. $^{8}$

The deliberate safeguards are hereby elaborated which shall bolster the desired intervention. Moreover, clinicians need to inculcate the wary precautions in daily routine. The outbreak has already affected 207 countries, while active cases have galloped to over a million with the concatenation of rapid spread originating in Wuhan city, China. To make the matters worse, despite the
1Department of Periodontics, ITS Dental College and Research Centre, Greater Noida, Uttra Pradesh, India

${ }^{2}$ Department of Prosthodontics, ITS Dental College and Research Centre, Greater Noida, Uttra Pradesh, India

Corresponding Author: Sumit Munjal, Department of Periodontics, ITS Dental College and Research Centre, Greater Noida, Uttra Pradesh, India, Phone: +91 9910259040, e-mail: ssmunjal@yahoo.co.in

How to cite this article: Munjal S, Munjal S. Pandemic COVID-19 and Its Implications in Dentistry: Overt Actions to Deal with Covert Threat. J Oral Health Comm Dent 2021;15(2):92-97.

Source of support: Nil

Conflict of interest: None

comparison on instigated drug trials, no specific antiviral therapy has been approved for the treatment of infection by human CoVs at present. An attempt to clear the fog of uncertainty to a maximum in this regard is by enlarging the purpose of this review.

\section{Materials and Methods}

Electronic data extraction was carried out in Google, PubMed, Wikipedia and Wiley Online Library, Indian Council of Medical Research, and World Health Organization (WHO) website between 2019 and 2020 present using appropriate keywords. Out of a total of 94 publications strategically obtained, 49 full articles were selected against the backdrop of the study title. All the pertinent information was compiled pursuing the PRISMA guidelines ${ }^{9}$ partially for transparent reporting. We had even activated additional 


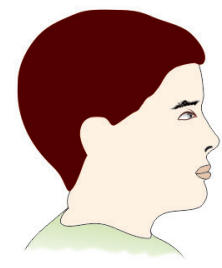

Infected patient

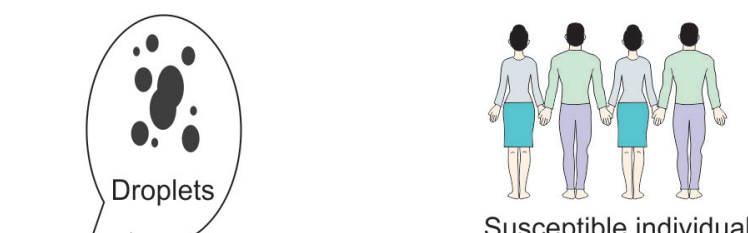

Susceptible individuals

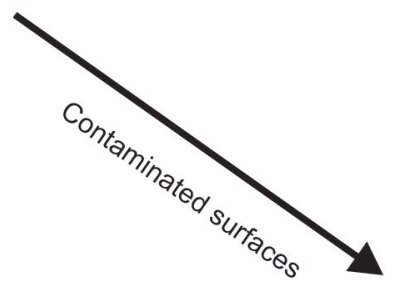

Fig. 1: Flow diagram

filters, namely the Reviews and English language. The exclusion was conference proceedings, video recordings, and all the material other than journals' full-texts (Fig. 1). Additionally, a manual search and queries with cross-reference were performed; nonetheless, 32 studies were included in the end.

\section{Diagnosis and Routes of Transmission in Dental Clinics}

First of all, clinicians should be able to identify a suspected case of COVID-19 as we are among the healthcare workers bearing the brunt of the situation. The patients present with fever, cough, and myalgia or fatigue with abnormal chest $\mathrm{CT}^{10}$ and less commonly sputum production, headache, hemoptysis, and diarrhea., ${ }^{8,10}$ It is noteworthy that the timing of the outbreak matches the seasonal flu, presenting the same symptoms and thus a confounder in proper diagnosis. Next, the symptoms need necessarily be confirmed by detailed investigations. Due to the characteristics of dental settings, the risk of cross infections may be high between dental practitioners and patients. ${ }^{8}$

Currently, transmission routes are still to be determined, but common ones include direct transmission (cough, sneeze, and droplet inhalation transmission) and contact transmission (contact with oral, nasal, and eye mucous membranes) (Fig. 2) ${ }^{8}$. The aerosol generation in dental devices ${ }^{8,11}$ has been studied as conversational airborne and longer-range transmission routes (over several meters). The former can then settle on surfaces (fomites) from where they can be touched and carried on hands leading to further self-inoculation routes of transmission. The incubation period ranges from 0 to 24 days; therefore, transmission can occur before any symptoms are apparent. ${ }^{8,12}$ The pathogen was recently identified in the saliva of the infected patients. Saliva can have a pivotal role in the human-to-human transmission and ${ }^{8,13}$ is studied as a useful diagnostic biomarker. ${ }^{14}$

\section{Infection Control in Dental Clinic}

Obtaining a thorough international travel and health history is vital, and dental offices should consider postponing nonemergency dental care to patients within 14 days of the travel. In the unlikely event of providing dental care to suspected or confirmed cases of COVID-19, dentists must be cognizant of the following:

\section{Standard Precautions}

- Optimal hand hygiene: Even though a routine prerequisite for the dental practice, but compliance needs to be reinforced before and after procedures among the staff sans touching their own eyes, mouth, and nose.

- Preoperational antimicrobial mouthrinse: The chlorhexidine is not effective as per the Guideline for the Diagnosis and Treatment of Novel Coronavirus Pneumonia (the 5th edition) released by the National Health Commission of the People's Republic of China, but SARS viruses are susceptible to povidone mouthrinse and the latter might reduce the load of coronaviruses in saliva. ${ }^{8}$

- Use of a barrier for protection: Although a patient with COVID-19 infection is not expected to be treated in the dental clinic, in the unlikely event that this does occur, the three level protection namely primary, secondary, and tertiary protection are recommended for specific situations.

- Primary protection consists of standard protection for staff in clinical settings, that is, wearing a disposable working cap, 
The type of PPE used will vary based on the level of precautions required, such as standard and contact, droplet or airborne infection isolation precautions. The procedure for putting on and removing PPE should be tailored to the specific type of PPE.

\section{GOWN}

- Fully cover torso from neck to knees, arms

to end of wrists, and wrap around the back

- Fasten in back of neck and waist
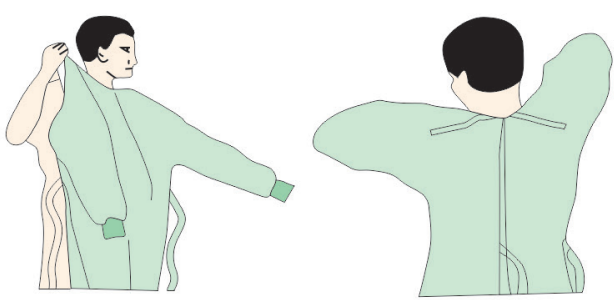

\section{MASK OR RESPIRATOR}

- Secure ties or elastic bands at middle

of head and neck

- Fit flexible band to nose bridge

- Fit snug to face and below chin

- Fit-check respirator
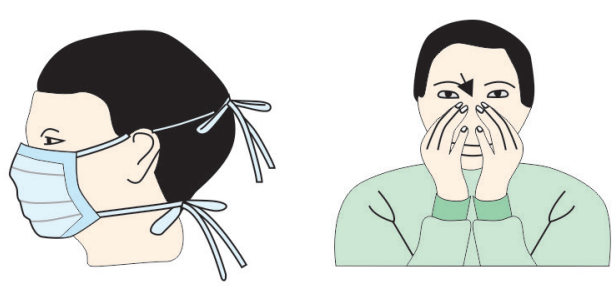

3. GOGGLES OR FACE SHIELD

- Place over face and eyes and adjust to fit
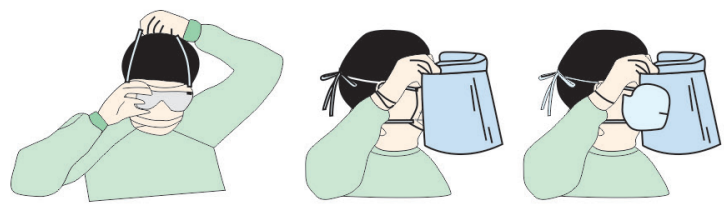

4.GLOVES

- Extend to cover wrist of isolation gown

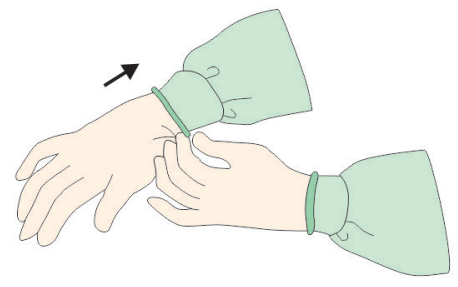

Fig. 2: Transmission routes of COVID-19 in dental clinics and hospitals

disposable surgical mask, and working clothes (white coat), using protective goggles or face shield, and disposable latex gloves or nitrile gloves if necessary.

- Secondary protection refers to advanced protection for dental professionals, that is, wearing a disposable doctor cap, disposable surgical mask, protective goggles, face shield, and working clothes (white coat) with disposable isolation clothing or surgical clothes outside, and disposable latex gloves.

- Tertiary protection infers the strengthened protection during suspected or confirmed patient contact.

The centers for disease control and prevention (CDC) recommends donning (Fig. 3) and doffing (Flowchart 1) personal protective equipment (PPE) for treating COVID-19 patients as is illustrated.

- Scrupulous isolation: Along with disposable (single use) devices such as mouth mirror, syringes, and blood pressure cuff to prevent cross-contamination, practitioners should use a rubber dam to minimize splatter generation. Anti-retraction dental
Flowchart 1: Doffing of PPE for treating COVID-19 patients

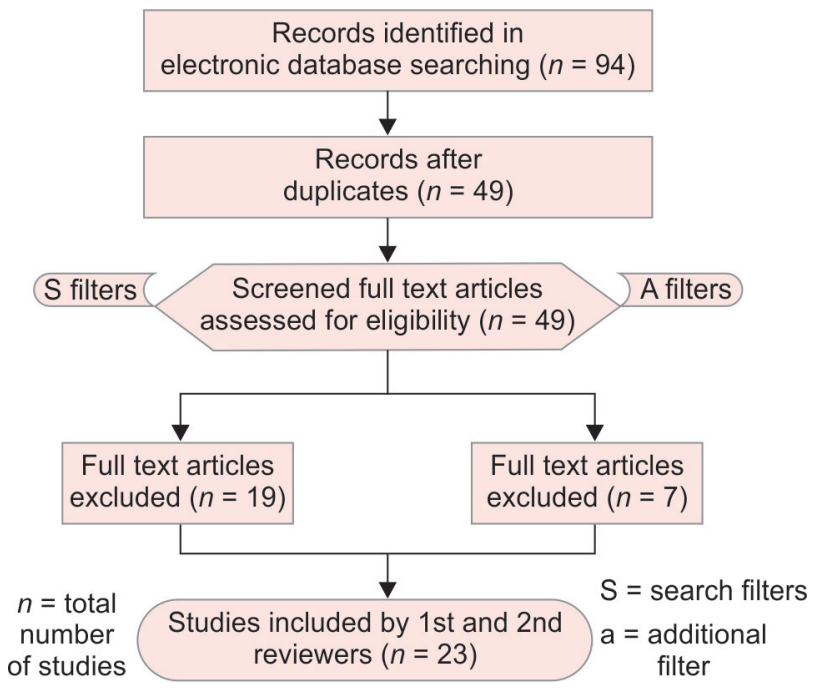




\section{GOWN AND GLOVES}

- Gown front and sleeves and the outside of gloves are contaminated!

- If your hands get contaminated during gown or glove removal,

immediately wash your hands or use an alcohol-based hand sanitizer

- Grasp the gown in the front and pull away from your body so

that the ties break, touching outside of gown only with gloved hands

- While removing the gown, fold or roll the gown inside-out into a bundle

- As you are removing the gown, peel off your gloves at the

same time, only touching the inside of the gloves and gown

with your bare hands. Place the gown and gloves into a waste container
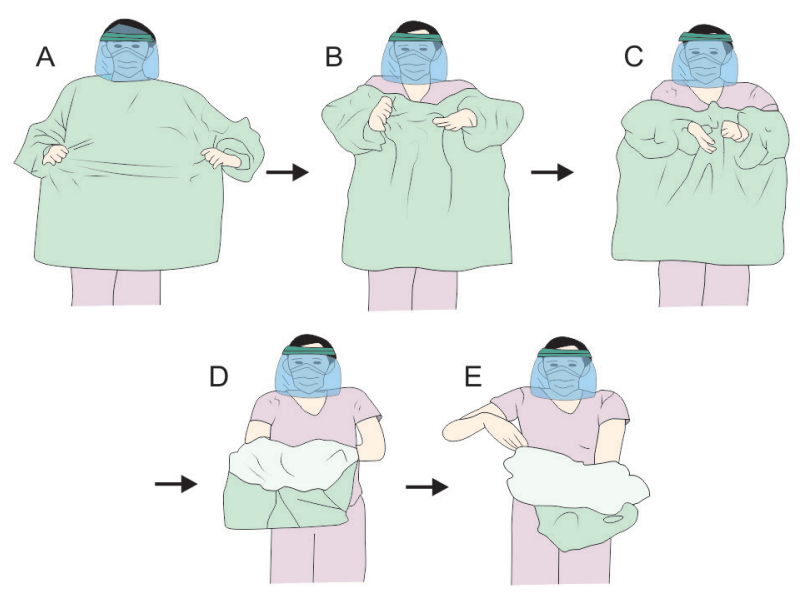

\section{GOGGLES OR FACE SHIELD}

- Outside of goggles or face shield are contaminated I

- If your hands get contaminated during goggle or face shield removal,

immediately wash your hands or use an alcohol-based hand sanitizer

- Remove goggles or face shield from the back by lifting head band and

without touching the front of the goggles or face shield

- If the item is reusable, place in designated receptacle for

reprocessing. Otherwise, discard in a waste container

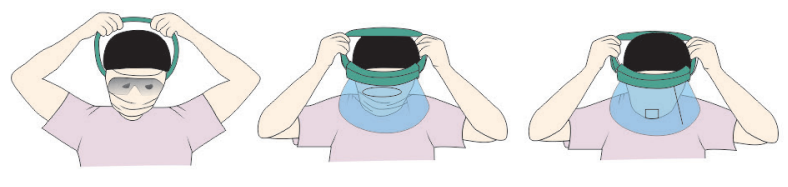

\section{MASK OR RESPIRATOR}

- Front of mask/respirator is contaminated - DO NOT TOUCH I

- If your hands get contaminated during mask/respirator removal,

immediately wash your hands or use an alcohol-based hand sanitizer

- Grasp bottom ties or elastics of the mask/respirator, then the ones at

the top, and remove without touching the front

- Discard in a waste container
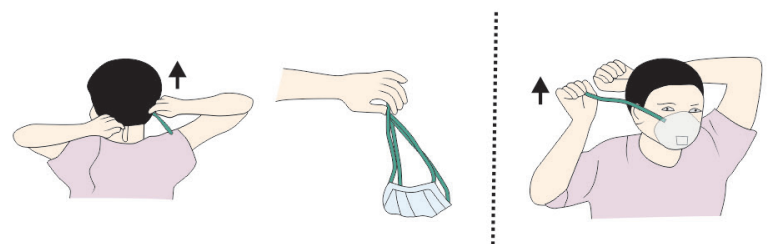

\section{WASH HANDS OR USE AN}

ALCOHOL-BASED HAND SANITIZER

IMMEDIATELY AFTER REMOVING

ALL PPE
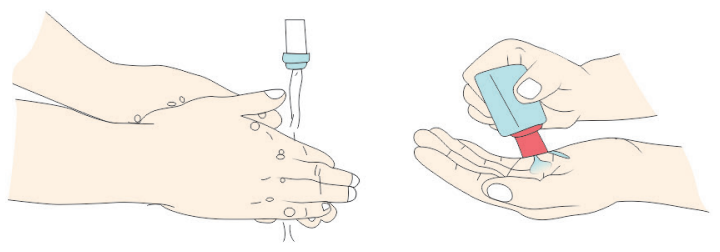

Fig. 3: Donning of PPE for treating COVID-19 patients

handpiece with specially designed anti-retractive valves or other anti-reflux designs is an extra preventive measure for cross infection. ${ }^{8}$ The X-rays of choice are extraoral imaging such as panoramic radiograph or cone beam computed tomography in order to avoid gag reflex or cough that may occur with intraoral imaging.

- Punctilious disinfection: The reusable instrument and items should be pretreated, cleaned, sterilized, and properly stored in accordance with the CDC guidelines. Both the chamber and public area, including door handles, chairs, and desks, ought to be strictly disinfected; the elevator is not an exception. People taking elevators should wear masks correctly and avoid direct contact with buttons and other objects.

- Waste management: The waste (including PPE) should be transported to the temporary storage area of the medical institute timely. General waste should be segregated from infectious in clearly marked bins, bagged and tied, and disposed of as general municipal waste (as per the Biomedical
Waste Management guidelines 2016, laid down by the Central Pollution Control Board, India). Infectious waste produced during patient care, including those with confirmed COVID-19 infection (e.g., sharps, bandages, and pathological waste), should be collected safely in color-coded bags (tied with gooseneck ligation), clearly marked lined containers, and sharp boxes.

\section{Management}

Patients are managed following WHO guidelines, wherein oxygen and intravenous fluids are recommended (the targets are $\mathrm{SpO}_{2} \geq 90 \%$ ) to mild and severe ones. ${ }^{15}$ The drug trials by nonsteroidal anti-inflammatory drugs, glucocorticoids, IL-6 antagonist, antiviral agents, and hydroxychloroquine ${ }^{15-17}$ are progressive in the pharmacological indagation.

Favipiravir, also known as T-705, is an antiviral drug developed in Japan in 2002 that inhibits viral RNA-dependent RNA polymerase, preventing the virus from replication. ${ }^{18}$ It is known now that the 
endogenous interferons are produced in response to viral infections and modulate the immune response by exerting antiviral activity. The coronaviruses are highly susceptible to interferon treatment in vitro. ${ }^{19}$

Combination of western and Chinese treatment by lopinavir and Shufeng jiedu capsule gained significant improvements in pneumonia-associated symptoms in Shangai Public Health Clinical Centre, China. ${ }^{20}$ But when severe, as in the septic shock, hypotension is a substantial sign and international guidelines are followed. ${ }^{21}$ Furthermore, pulmonary fibrosis in patients recovered from SARS-CoV-2 infections is an issue that urgently needs to be addressed. ${ }^{22}$ As of today, vaccines are the most effective strategy since they are more cost-effective than treatment. ${ }^{23}$

\section{Discussion}

The mankind has hitherto seen three worldwide pandemics since 2000, HINI (Swine flu) in 2009, Ebola virus in 2013, and now COVID-19. The latter shall definitely be described as a black swan in history. The lessons were seldom learned even upon the casualties of apocalyptic proportions in China. Italy is the second most affected country. ${ }^{24} \mathrm{WHO}$ declared a public health emergency of international concern over this global pneumonia outbreak on January $30,2020{ }^{3}$ We are going through a rapidly changing situation that has not been experienced before. The real-time polymerase chain reaction test and point-of-care molecular diagnostic assays are universal diagnostics. The rapid antibody test has also been suggested as a supplementary test, which is only good for surveillance as the results come after 7-10 days of the pandemic infection. ${ }^{25}$

COVID-19 indicator list is growing larger now than before. It seems we now have to suspect everything. Along with the general flu, new symptoms that have been included are chills, loss of smell and taste, mouth rashe, ${ }^{26}$ and the presence of enanthem (oral cavity lesions). ${ }^{27}$ The limitations do exist owing to the ever-changing manifestations due to virus mutations. When the global focus has mostly been on testing, finding a cure, and preventing transmission, people are going through a myriad of psychological problems in adjusting to the current lifestyles and fear of the disease. In the anticipation of mise en scene, India has launched "ArogyaSetu" mobile application, which is an informative tool to spread awareness regarding COVID-19 among the common public. ${ }^{28}$ The app utilizes the Bluetooth and GPS tracker of the mobile phones to identify if someone has crossed the paths with the disease-ridden individual by completely scanning the user's location as well the database of the confirmed cases in the country. The details of the infected individuals are kept confidential so that there is no fear of social rejection or stigma among the people.

Providing psychological first aid and counseling are quintessential during an epidemic. Recently, a cross-sectional study with a considerable sample size was done to assess the initial impact of COVID-19 on fears, worries, and impairment in sleep. The factors that predicted higher impact were younger age, being female, and having a known physical comorbidity. ${ }^{29}$ Even though the limitations were observed in the above as it was conducted during a period of lockdown, yet it highlighted a need for considering mental health issues by the policymakers. It helps in reducing the psychological distress and promoting adaptive coping strategies to deal with the situation. ${ }^{30}$

The standard operating procedures have been formulated for all healthcare locations. But, there is still a long way to go to have the curse of travail been lifted from the human soul.
Best practices for safely managing healthcare waste should be followed. This waste should be treated, preferably on-site, and then safely disposed of. Preferred treatment options are high temperature, dual-chamber incineration, or autoclaving. ${ }^{31}$ Last but not the least, obtaining written consent from each and every patient in dental set-up needs is a prerequisite for treatment services. The declaration of the travel history, COVID-19 symptoms, and awareness on cough etiquette are the mandatory inclusions in patients' signed statements. Above all, dental health-care providers ought to be trained adequately and simultaneously impart the same knowledge to their staff until we zap the force majeure.

\section{CONCLUSION}

The world is woefully facing the challenge of SARS-CoV-2 onslaught, and the cases are skyrocketing with each passing day. The current dogma in crisis is aptly referred to as containment measures that are isolation in the healthcare milieu, quarantine for the exposed patients, and social distancing for the public as a whole.

\section{ORCID}

Sumit Munjal @ https://orcid.org/0000-0001-5990-2832

\section{References}

1. Morse SS. Factors in the emergence of infectious diseases. Emerg Infect Dis 1995;1(1):7-15. DOI: 10.3201/eid0101.950102.

2. Jones $\mathrm{KE}$, Patel NG, Levy MA, et al. Global trends in emerging infectious disease. Nature 2008;451(7181):990-993. DOI: 10.1038/ nature06536.

3. Sohrabi C, Alsafi Z, O' Neill N, et al. World Health Organisation declares emergency: a review of the 2019 novel coronavirus (covid 19). Int J Surg 2020;76:71-76. DOI: 10.1016/j.jijsu.2020.02.034.

4. Yu F, Du L, Ojcius DM, et al. Measures for diagnosing and treating infections by a novel coronavirus responsible for a pneumonia outbreak originating in Wuhan, China.Microbes Infect 2020;22(2): 74-79. DOI: 10.1016/j.micinf.2020.01.003.

5. Hui DSC, Zumla A. Severe acute respiratory syndrome: historical, epidemiologic, and clinical features. Infect Dis Clin North Am 2019;33(4):869-889. DOI: 10.1016/j.idc.2019.07.001.

6. Holmes KV. SARS-associated coronavirus. N Engl J Med 2003;348: 1948-1951. DOI: 10.1056/NEJMp030078.

7. Falsey AR, Walsh EE. Novel coronavirus and severe acute respiratory syndrome. Lancet 2003;361(9366):1312-1313. DOI: 10.1016/S01406736(03)13084-X.

8. Peng X, Xu X, Li Y, et al. Transmission routes of 2019-nCoV and controls in dental practice Int J Oral Sci 2020;12(1):9. DOI: 10.1038/s41368-0200075-9.

9. Liberati A, Altman DG, Telzlaff J, et al. The PRISMA statement for reporting systemic reviews and meta-analysis of studies that evaluate healthcare interventions: Explanation and elaboration. BMJ 2009;339:b2700. DOI: 10.1136/bmj.b2700.

10. Zhu N, Zhang $D$, Wang $W$, et al. A novel coronavirus from patients with pneumonia in China 2019. N Engl J Med 2020;382(8):727-733. DOI: 10.1056/NEJMoa2001017.

11. Tellier R, Li Y, Cowling BJ, et al. Recognition of aerosol transmission of infectious agents: a commentary. BMC Infect Dis 2019;19:101. DOI: 10.1186/s12879-019-3707-y.

12. Rothe C, Schunk M, Sothmann P, et al. Transmission of 2019-n Cov infection from an asymptomatic contact in Germany. N Engl J Med 2020;382(10):970-971. DOI: 10.1056/NEJMc2001468.

13. Sabino-Silva R, Jardin ACG, Siqueira WL. Coronavirus COVID-19 impacts to and potential salivary diagnosis. Clin Oral Investig 2020;24(4):1619-1621. DOI: 10.1007/s00784-020-03248-x. 
14. Zuanazzi D, Arts EJ, Jorge PK, et al. Postnatal identification of Zika virus peptides from saliva. J Dent Res 2017;96(10):1078-1084. DOI: $10.1177 / 0022034517723325$.

15. Cheng ZJ, Shan J. 2019 novel coronavirus: where we are and what we know. Infection 2020;48(2):155-163. DOI: 10.1007/s15010-02001401-y.

16. Cortegiani A, Ingoglia G, Ippolito M, et al. A systematic review on the efficacy and safety of chloroquine for the treatment of COVID-19. J Crit Care DOI: 10.1016/j.jcrc.2020.03.005. Epub 2020 Mar 10. PMID: 32173110; PMCID: PMC7270792.

17. Zhang $W$, Zhao $Y$, Zhang $F$, et al. The use of anti-inflammatory drugs in the treatment of people with severe coronavirus disease 2019 (COVID-19): the perspectives of clinical immunologists from China. Clin Immunol 2020;214:108393. DOI: 10.1016/j.clim.2020.108393.

18. Furuta $Y$, Komeno T, Nakamura T. Favipiravir (T-705), a broad-spectrum inhibitor of viralRNA polymerase. Proc Jpn Acad Ser B Phys Biol Sci 2017;93(7):449-463. DOI: 10.2183/pjab.93.027.

19. Gralinski LE, Baric RS. Molecular pathology of emerging coronavirus infections. J Pathol 2015;235(2):185-195. DOI: 10.1002/path.4454.

20. Guo YR, Cao QD, Hong ZS, et al. The origin, transmission and clinical therapies on coronavirus disease 2019 (COVID-19) outbreak - an update on the status. Mil Med Res 2020;7(1):11. DOI: 10.1186/s40779020-00240-0.

21. Rhodes A, Evans Le, Ahazzani W, et al. Surviving sepsis campaign: international guidelines for management of sepsis and septic shock. Intensive Care Med 2017;43(3):304-377. DOI: 10.1007/s00134-0174683-6.

22. Sun P, Lu X, Xu C, et al. Understanding of COVID-19 based on current evidence. J Med Virol 2020;92(6):548-551. DOI: 10.1002/jmv. 25722.

23. Ahn DG, Shin HJ, Kim MH, et al. Current status of epidemiology, diagnosis, therapeutics and vaccines for novel coronavirus disease
2019 (COVID-19). J Microbiol Biotechnol 2020;30(3):313-324. DOI: 10.4014/jmb.2003.03011.

24. Remuzzi A, Remuzzi G. COVID-19 and Italy: what next? Lancet 2020;395(10231):1225-1228. DOI: 10.1016/S0140-6736(20)30627-9.

25. COVID 19-Indian council of medical research/Government of India. Available from: https://icmr.nic.in/content/covid-19 [Accessed April 17, 2020].

26. Jimenez-Cauhe J, Ortega-Quijano D, de Perosanz-Lobo D, et al. Enanthem in patients with COVID-19 and skin rash. JAMA Dermatol 2020;156(10):1134-1136. DOI: 10.1001/jamadermatol.2020.2550.

27. Drago F, Paolino S, Rebora A, et al. The challenge of diagnosing atypical exanthems: a clinico-laboratory study. J Am Acad Dermatol 2012;67(6):1282-1288. DOI: 10.1016/j.jaad.2012.04.014.

28. Government of India/Aarogya setu mobile app. Available from: https://www.mygov.in/aarogya-setu-app [Accessed May 25, 2020].

29. Varshney M, Parel JT, Raizada N, et al. Initial psychological impact of COVID-19 and its correlates in Indian Community: an online (FEEL-COVID) survey. PLoS One 2020;15(5):e0233874. DOI: 10.1371/ journal.pone.0233874.

30. Patel A, Jernigan DB; 2019-nCoV CDC Response Team. Initial Public Health Response and Interim Clinical Guidance for the 2019 Novel Coronavirus Outbreak - United States, December 31, 2019-February 4, 2020. MMWR Morb Mortal Wkly Rep. 2020 Feb 7;69(5):140-146. DOI: 10.15585/mmwr.mm6905e1. Erratum in: MMWR Morb Mortal Wkly Rep. 2020 Feb 14;69(6):173. PMID: 32027631; PMCID: PMC7004396.

31. World Health Organization. Water, sanitation, hygiene, and waste management for the COVID-19 virus: interim guidance. July 29, 2020. Available from: https://apps.who.int/iris/rest/bitstreams/1289390/ retrieve. 